\title{
Down-regulation of PM20D1 is associated with clinical outcomes and prognosis of pregnant patients with diabetes mellitus
}

\author{
Jie Hou, Xiaohong Chen, Jianqing Xia, Liemin Zhang
}

Adult Internal Department, Maternal and Child Health Hospital of Hubei Provincial, Wuhan, China

Submitted: 14 April 2021; Accepted: 29 April 2021

Online publication: 9 May 2021

Arch Med Sci 2022

DOI: https://doi.org/10.5114/aoms/136284

Copyright $\odot 2022$ Termedia \& Banach

\section{Abstract}

Introduction: The present study aimed to investigate the role of peptidase M20 domain containing 1 (PM20D1) in gestational diabetes mellitus (GDM). Material and methods: This observational study included 189 cases of GDM patients who came to our department between March 2018 and December 2019. Additionally, 100 healthy pregnant individuals who came to physical examination were included as healthy controls during the same period. Western blotting was used to determine the expression of PM20D1 at gestational age 24-28 weeks and gestational age 37-40 weeks. Serum inflammatory factors of C-reactive protein (CRP), interleukin (IL)-1 $\beta$, IL-6, and tumor necrosis factor (TNF)- $\alpha$, as well as leptin and adiponectin, were all measured by ELISA. The GDM related indices were also measured.

Results: The expression of PM20D1 was markedly decreased in GDM patients compared with the healthy controls. Patients in the third trimester of pregnancy (gestational age 37-40 weeks) showed significantly lower expression of PM20D1 than patients in the second trimester of pregnancy (gestational age 24-28 weeks). Serum levels of CRP, IL-1 $\beta$, IL-6, TNF- $\alpha$ and leptin were remarkably higher and levels of adiponectin were markedly lower in GDM patients at both the second and third trimester of pregnancy. Also, levels of CRP, IL-1 $\beta$, IL-6, TNF- $\alpha$ and leptin in GDM patients were the highest at the third trimester of pregnancy. Pearson's analysis showed that PM20D1 was negatively correlated with IL-1 $\beta$, IL- 6 and leptin and was positively correlated with adiponectin. At the second trimester of pregnancy, patients with lower expression of PM20D1 showed remarkably higher levels of HOMA-IR, fasting insulin, FBG, OGTT-1hPG, OGTT-2hPG, TG and LDL-C, and showed markedly lower levels of HDL-C. Down-regulated PM20D1 predicted poor pregnancy outcomes.

Conclusions: Reduced PM20D1 was associated with patients' clinical outcomes and pregnancy outcomes in GDM.

Key words: PM20D1, gestational diabetes mellitus, inflammatory factors, pregnancy outcomes.

\section{Introduction}

Gestational diabetes mellitus (GDM), which is defined as diabetes diagnosed in the second or third trimester of pregnancy that was not clearly overt diabetes prior to gestation according to the American Diabetes Association (ADA) [1], can cause a series of diseases including metabolic

\author{
Corresponding author: \\ Liemin Zhang \\ Adult Internal Department \\ Maternal and Child Health \\ Hospital of Hubei Provincial \\ Wuhan, 430070, China \\ E-mail: zleim14@21cn.com
}


disorders, fetal malformation, polyhydramnios, spontaneous abortion, fetal distress, stillbirth, ketoacidosis, neonatal hypoglycemia, hypocalcemia, hyperbilirubinemia, and postpartum development of type 2 diabetes [2-5]. It is reported that the prevalence of GDM is more than $10 \%$ in all pregnant women [6, 7]. However, mechanisms for how GDM occurs are still unclear.

Peptidase M20 domain containing 1 (PM20D1) is a newly identified secreted enzyme enriched in UCP1+ vs. UCP1- adipocytes [8]. It is reported that PM20D1 participates in many diseases and bioprocesses. Sanchez et al. found that PM20D1 was a quantitative trait locus associated with Alzheimer's disease [9]. Long et al. considered that ablation of PM20D1 might regulate the metabolism and nociception of N-acyl amino acid [10]. It was also found that PM20D1 might have a non-additive biochemical engagement for $\mathrm{N}$-acyl amino acid metabolism and underscore enzymatic division [11]. Another study also found that methylation of PM20D1 was elevated in pregnant women with asthma [12]. However, up to now, no study has focused on PM20D1 in GDM.

The present study aimed to investigate the role of PM20D1 in GDM. This research might provide better understanding and some new research targets for PM20D1 in GDM development.

\section{Material and methods}

\section{Patients}

This prospective observational study included 189 cases of GDM patients who came to our department between March 2018 and December 2019. All gestational patients who met the inclusion criteria were consecutively enrolled and were diagnosed with diabetes. The diagnosis of GDM was according to the World Health Organization (WHO) and the International Association of Diabetes in Pregnancy Study Groups (IADPSG) diagnostic criteria [13]. One of the following could be diagnosed as GDM: fasting blood glucose (FBG) $\geq 5.1 \mathrm{mmol} / \mathrm{l}, 75 \mathrm{~g}$ oral glucose tolerance test (OGTT) 1-hour plasma glucose (OGTT-1hPG) $\geq 10.0$ $\mathrm{mmol} / \mathrm{l}$, or OGTT-2hPG $\geq 8.5 \mathrm{mmol} / \mathrm{l}$ in gestational patients with gestational age 24-28 weeks. The following patients were excluded: a) patients with severe infections, chronic infections or asthma; b) patients with liver, renal or cardiovascular diseases such as HBV, renal failure or coronary heart disease; c) patients who had diabetes before pregnancy. Additionally, 100 healthy pregnant individuals who came to physical examination were included as healthy controls during the same period. All healthy pregnant individuals were confirmed to have no related diseases such GDM, cardiovascular diseases, other metabolic diseases, liver or renal dysfunctions. Written informed consent was obtained from all patients. The present study was approved by the ethics committee of the Maternal and Child Health Hospital of Hubei Provincial.

\section{Western blotting for PM20D1}

The patients' blood samples were collected at gestational age 24-28 weeks, as well as gestational age 37-40 weeks. The serum levels of PM20D1 were determined by western blotting. Proteins were extracted from the samples by radio-immunoprecipitation assay (RIPA) buffer (Vazyme Biotec Co., LTD, Nanjing, China). Protein contents were determined using protein assay reagent from BioRad (Hercules, CA, USA). The serum albumin was depleted by using Bio-Rad DEAE Affi-Gel Blue (BioRad). The extracted proteins $(30 \mu \mathrm{g})$ were loaded on $10 \%$ SDS-PAGE and transferred to PVDF membranes, following by incubation with a primary anti-PM20D1 antibody (LS-C205961, LifeSpan Biosciences) followed with a conjugated secondary antibody (LS-C69961, LifeSpan Biosciences). The films were scanned by the EasySee Western Blot Kit (Beijing TransGen Biotech, Beijing, China). Relative protein expression was quantified using Image-Pro Plus software (version 6.0; Media Cybernetics, Inc., Rockville, MD, USA). GAPDH was used as the reference protein.

\section{Measurement of serum inflammatory factors}

Inflammatory markers of C-reactive protein (CRP), interleukin (IL)-1 $\beta$, IL-6, and tumor necrosis factor (TNF)- $\alpha$, as well as leptin and adiponectin, were all measured by commercial ELISA kits according to the manufacturers' instructions (CRP ab260058 Abcam sensitivity $5.36 \mathrm{pg} / \mathrm{ml}$ range $18.75 \mathrm{pg} / \mathrm{ml}-1200 \mathrm{pg} / \mathrm{ml}$, IL-1 $\beta$ ab214025 Abcam sensitivity $5.64 \mathrm{pg} / \mathrm{ml}$ range $14.06 \mathrm{pg} / \mathrm{ml}$ - 900 pg/ml, IL-6 LS-F25919 LifeSpan Biosciences sensitivity $5.36 \mathrm{pg} / \mathrm{ml}$ range $18.75 \mathrm{pg} / \mathrm{ml}$ - 1200 pg/ml, TNF- $\alpha$ ab181421 Abcam sensitivity $4.32 \mathrm{pg} / \mathrm{ml}$ range $15.63 \mathrm{pg} / \mathrm{ml}-1000 \mathrm{pg} / \mathrm{ml}$, leptin ab179884 Abcam, sensitivity $4.65 \mathrm{pg} / \mathrm{ml}$ range $15.63 \mathrm{pg} / \mathrm{ml}-1000 \mathrm{pg} / \mathrm{ml}$ and adiponectin ab99968 Abcam sensitivity $<25 \mathrm{pg} / \mathrm{ml}$ range not mentioned).

\section{Clinical outcomes and follow-up}

Patients' characteristics and demographic data were collected. The total cholesterol (TC), triglyceride (TG), high-density lipoprotein cholesterol (HDL-C) and low-density lipoprotein cholesterol (LDL-C), as well as the levels of FBG, OGTT-1hPG, OGTT-2hPG and fasting insulin, were measured by an automatic biochemical analyzer (Beckman Coulter AU5800). The insu- 
lin resistance index (HOMA-IR) was calculated and recorded by: (fasting insulin $(\mathrm{mU} / \mathrm{l}) \times \mathrm{FBG}$ $(\mathrm{mmol} / \mathrm{l})) / 22.5$. All patients were followed up until delivery.

\section{Statistical analysis}

All experiments were conducted in triplicate. The distribution of the data was analyzed by the Kolmogorov-Smirnov method. The data distributed normally were expressed as mean $\pm S D, \chi^{2}$ test was used to compare the rates and comparison between two groups was performed using the Student $t$-test. Comparison among three or more groups was performed by one-way analysis of variance (ANOVA) followed by the Tukey post hoc test. Logistic regression analysis was used for risk factors of poor pregnancy outcome. $P<0.05$ was considered as statistically significant. All calculations were made using SPSS 18.0.

\section{Results}

\section{Serum protein levels of PM20D1 were down-regulated in GDM patients}

The comparison of basic characteristics of the GDM patients and healthy controls is shown in Table I. No significant difference was found for age and BMI. However, the levels of HOMA-IR, fasting insulin, FBG, OGTT-1hPG, OGTT-2hPG, as well as TG and LDL-C were significantly higher in GDM patients and levels of HDL-C levels were remarkably lower in GDM patients compared with healthy controls (all $p<0.05$ ).

Table I. Basic characteristics of all GDM patients and healthy controls at 24-28 weeks after pregnancy when enrolled in the study

\begin{tabular}{|lccc|}
\hline Variables & GDM $(n=189)$ & Healthy controls $(n=100)$ & $P$-value \\
\hline Age $[$ year] & $25.97 \pm 3.62$ & $26.87 \pm 3.79$ & 0.051 \\
\hline BMI $\left[\mathrm{kg} / \mathrm{m}^{2}\right]$ & $23.84 \pm 2.77$ & $24.46 \pm 2.83$ & 0.072 \\
\hline HOMA-IR & $2.06 \pm 0.24$ & $1.26 \pm 0.14$ & $<0.001$ \\
\hline FBG $[\mathrm{mmol} / \mathrm{l}]$ & $5.38 \pm 0.52$ & $3.75 \pm 0.37$ & $<0.001$ \\
\hline OGTT-1hPG $[\mathrm{mmol} / \mathrm{l}]$ & $10.23 \pm 0.52$ & $8.22 \pm 0.42$ & $<0.001$ \\
\hline OGTT-2hPG $[\mathrm{mmol} / \mathrm{l}]$ & $8.89 \pm 0.36$ & $6.54 \pm 0.59$ & $<0.001$ \\
\hline Fasting insulin $[\mathrm{mU} / \mathrm{l}]$ & $14.26 \pm 2.01$ & $9.70 \pm 2.47$ & $<0.001$ \\
\hline TC $[\mathrm{mmol} / \mathrm{l}]$ & $5.86 \pm 0.75$ & $5.82 \pm 0.73$ & 0.696 \\
\hline TG $[\mathrm{mmol} / \mathrm{l}]$ & $3.02 \pm 0.49$ & $2.28 \pm 0.30$ & $<0.001$ \\
\hline HDL-C [mmol/l] & $2.03 \pm 0.14$ & $2.22 \pm 0.21$ & $<0.001$ \\
\hline LDL-C $[\mathrm{mmol} / \mathrm{l}]$ & $3.56 \pm 0.35$ & $3.23 \pm 0.31$ & $<0.001$ \\
\hline
\end{tabular}

$B M I$ - body mass index, FBG - fasting blood glucose, TC - total cholesterol, TG - triglyceride, HDL-C - high-density lipoprotein cholesterol, $L D L-C$ - low density lipoprotein cholesterol, OGTT - oral glucose tolerance test.

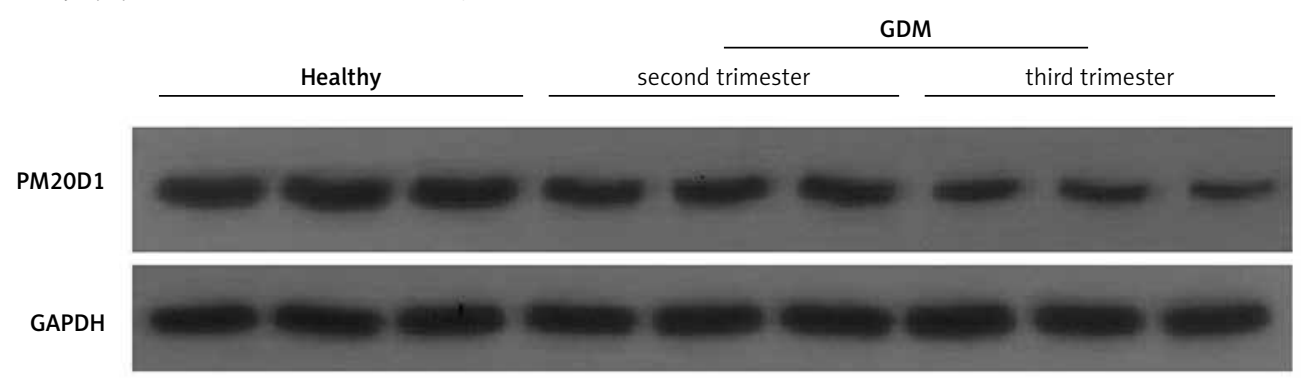

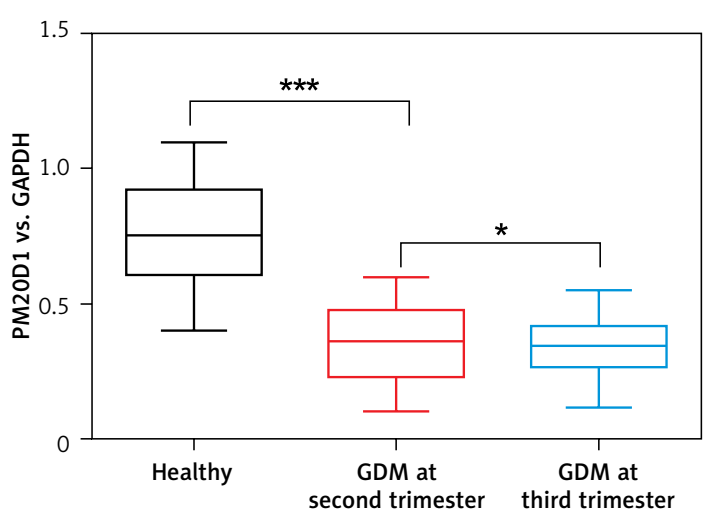

PM20D1

Figure 1. Serum protein levels of PM20D1 in all GDM patients, GDM patients of second trimester of pregnancy, GDM patients of third trimester of pregnancy, and healthy controls

PM20D1 - peptidase M20 domain containing 1, GDM - gestational diabetes mellitus. ${ }^{* * *} p<0.001,{ }^{*} p<0.05$. 
Then, western blotting was used for measurement of PM20D1 and its relative expression vs. GAPDH was calculated. As shown in Figure 1, the expression of PM20D1 was markedly lower in GDM patients compared with the healthy controls $(p<0.01)$. Also, patients in the third trimester of pregnancy (gestational age 37-40 weeks) showed significantly lower expression of PM20D1 than patients in the second trimester of pregnancy (gestational age $24-28$ weeks) $(p<0.05)$. These results indicated that PM20D1 expression was associated with GDM and the pregnancy time.

A

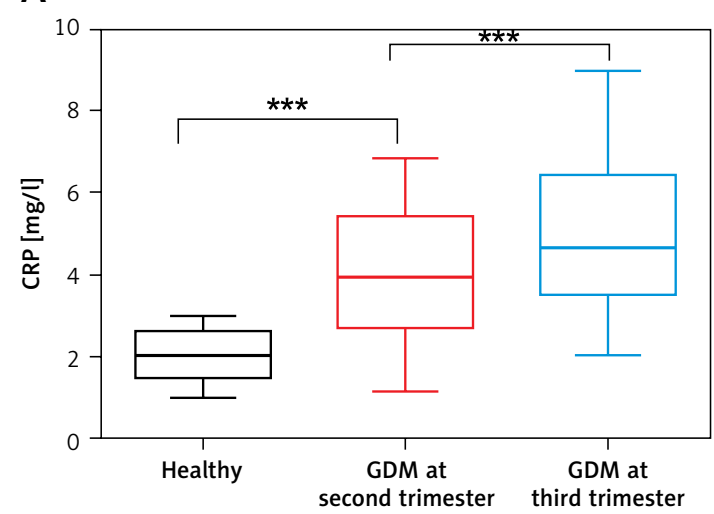

C

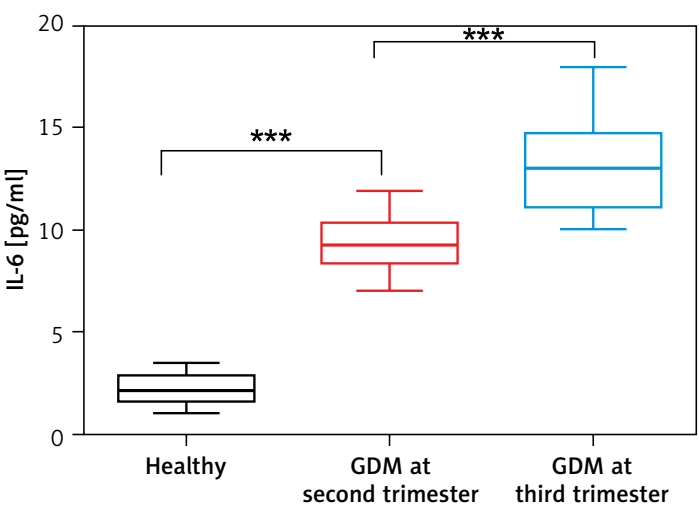

E

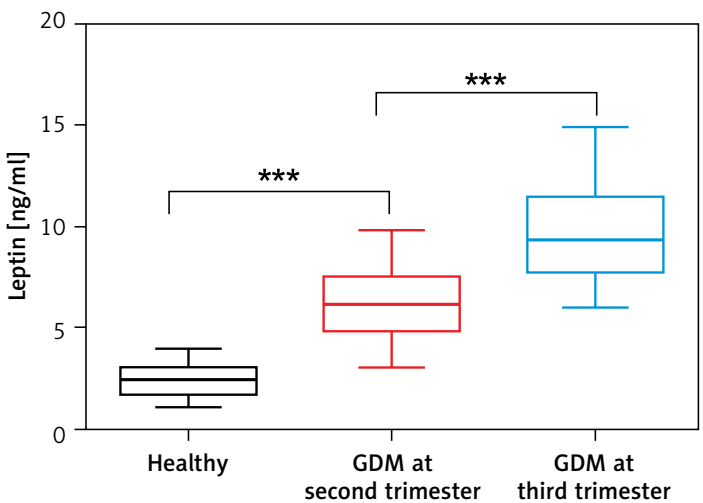

Serum levels of PM20D1 were correlated with inflammatory factors

To further investigate the role of PM20D1 in GDM, serum levels of inflammatory factors of CRP, IL-1 $\beta$, IL-6, TNF- $\alpha$, leptin and adiponectin were measured by ELISA at both 24-28 weeks and 37-40 weeks after pregnancy. It was found that factors of CRP, IL-1 $\beta$, IL- 6 , TNF- $\alpha$ and leptin were remarkably higher and levels of adiponectin were markedly lower in GDM patients at both the second and third trimester of pregnancy compared

B

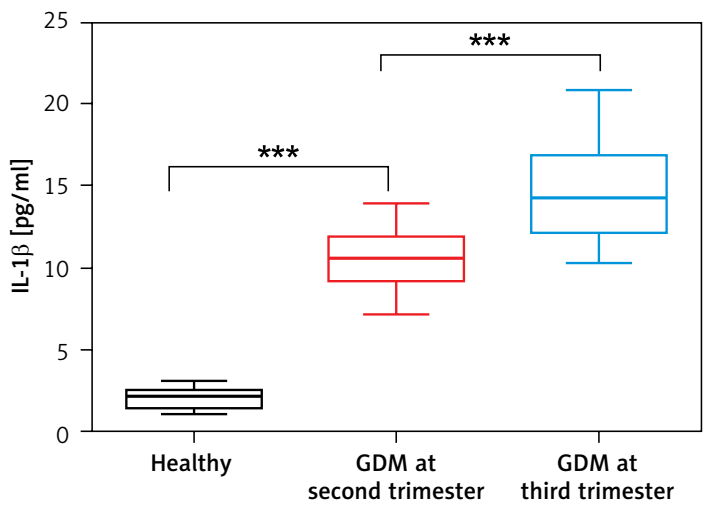

D

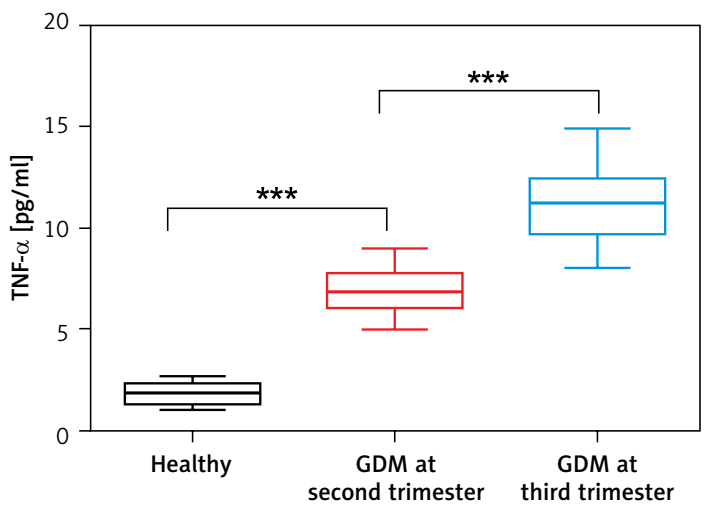

$\mathrm{F}$

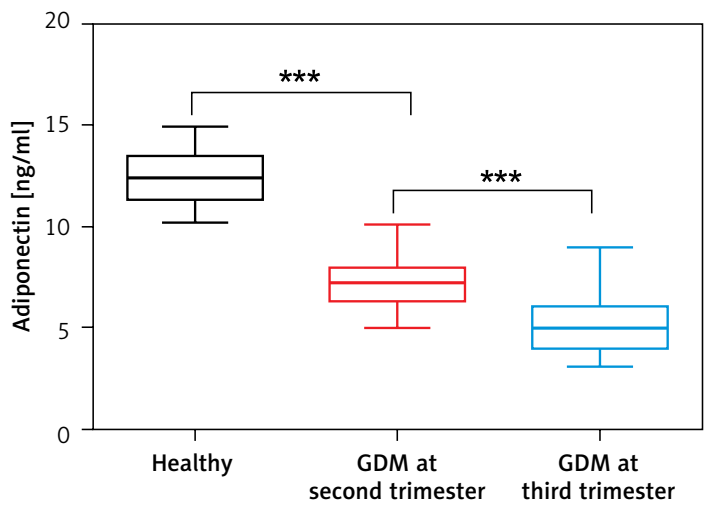

Figure 2. Serum protein levels of inflammatory factors of CRP, IL-1 $1 \beta$, IL-6, TNF- $\alpha$, leptin and adiponectin in all GDM patients, GDM patients of second trimester of pregnancy, GDM patients of third trimester of pregnancy, and healthy controls

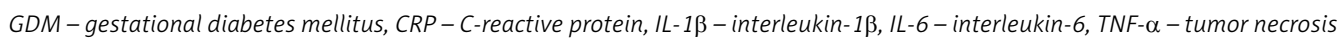
factor $\alpha .{ }^{* * *} p<0.001$. 
with the healthy controls ( $p<0.05$, Figure 2$)$. Also, levels of CRP, IL-1 $\beta$, IL-6, TNF- $\alpha$ and leptin were remarkably higher and levels of adiponectin were markedly lower in GDM patients at the third trimester of pregnancy than in GDM patients at the second trimester of pregnancy $(p<0.05)$. Furthermore, Pearson's analysis showed that PM20D1 was negatively correlated with IL-1 $\beta, I \mathrm{~L}-6$ and leptin and positively correlated with adiponectin at the second trimester of pregnancy and at the third trimester of pregnancy PM20D1 was also correlated with CRP, IL-6 and adiponectin (Table II).

\section{Serum levels of PM20D1 were correlated with diabetic-related indices}

Then, since the expression of PM20D1 was the lowest at the second trimester of pregnancy, all patients were divided into PM20D1 low or high expression groups according to mean relative value of PM20D1 (0.35) at 24-28 weeks of pregnancy. As shown in Table III, at the second trimester of pregnancy, patients with lower expression of PM20D1 showed remarkably higher levels of HOMA-IR, fasting insulin, FBG, OGTT-1hPG, OGTT2hPG, as well as TG and LDL-C, as well as markedly lower levels of HDL-C compared with patients with higher expression of PM20D1, suggesting that lower expression of PM20D1 might predict severer outcomes of GDM.

\section{Down-regulated PM20D1 was associated with poor pregnancy outcomes}

Finally, we analyzed the PM20D1 expression at 24-28 weeks after pregnancy and its relationship with the pregnancy outcomes. As shown in Table IV, it was found that the rates of premature,

Table II. Pearson's analysis for relative levels of PM20D1 and serum levels of inflammatory factors at second and third trimester of pregnancy

\begin{tabular}{|lccc|}
\hline & & \multicolumn{2}{c|}{ PM20D1 } \\
\cline { 2 - 4 } & & Second trimester of pregnancy & Third trimester of pregnancy \\
\hline \multirow{2}{*}{ CRP } & Pearson correlation & -0.507 & -0.149 \\
\cline { 2 - 4 } & $P$-value & $<0.001$ & 0.040 \\
\cline { 2 - 4 } & Pearson correlation & -0.807 & -0.111 \\
\hline \multirow{2}{*}{ IL-6 } & $P$-value & $<0.001$ & 0.127 \\
\cline { 2 - 4 } & Pearson correlation & -0.791 & -0.197 \\
\hline \multirow{2}{*}{ TNF- $\alpha$} & $P$-value & $<0.001$ & 0.006 \\
\cline { 2 - 4 } & Pearson correlation & -0.807 & 0.047 \\
\cline { 2 - 4 } Leptin & $P$-value & $<0.001$ & 0.515 \\
\cline { 2 - 4 } & Pearson correlation & 0.769 & -0.132 \\
\hline Adiponectin & $P$-value & $<0.001$ & 0.069 \\
\cline { 2 - 4 } & Pearson correlation & 0.769 & 0.175 \\
\cline { 2 - 4 } & $P$-value & $<0.001$ & 0.016 \\
\hline
\end{tabular}

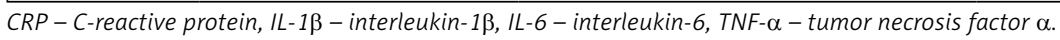

Table III. Diabetic-related indices in patients with different levels of PM20D1

\begin{tabular}{|c|c|c|c|}
\hline Variables & PM20D1 high $(n=99)$ & PM20D1 low $(n=90)$ & $P$-value \\
\hline Age [years] & $25.58 \pm 3.62$ & $26.41 \pm 3.59$ & 0.118 \\
\hline $\mathrm{BMI}\left[\mathrm{kg} / \mathrm{m}^{2}\right]$ & $23.55 \pm 2.83$ & $24.15 \pm 2.68$ & 0.144 \\
\hline HOMA-IR & $1.90 \pm 0.19$ & $2.25 \pm 0.14$ & $<0.001$ \\
\hline FBG [mmol/l] & $5.16 \pm 0.46$ & $5.63 \pm 0.45$ & $<0.001$ \\
\hline OGTT-1hPG [mmol/l] & $9.76 \pm 0.15$ & $10.76 \pm 0.15$ & $<0.001$ \\
\hline OGTT-2hPG [mmol/l] & $8.60 \pm 0.24$ & $9.20 \pm 0.16$ & $<0.001$ \\
\hline Fasting insulin [mU/l] & $13.16 \pm 1.78$ & $13.87 \pm 3.24$ & 0.061 \\
\hline $\mathrm{TC}[\mathrm{mmol} / \mathrm{l}]$ & $5.86 \pm 0.78$ & $5.86 \pm 0.72$ & 0.955 \\
\hline TG $[\mathrm{mmol} / \mathrm{l}]$ & $2.75 \pm 0.44$ & $3.32 \pm 0.33$ & $<0.001$ \\
\hline $\mathrm{HDL}-\mathrm{C}[\mathrm{mmol} / \mathrm{l}]$ & $2.15 \pm 0.08$ & $1.90 \pm 0.05$ & $<0.001$ \\
\hline LDL-C [mmol/l] & $3.37 \pm 0.29$ & $3.77 \pm 0.27$ & $<0.001$ \\
\hline
\end{tabular}

$B M I$ - body mass index, FBG - fasting blood glucose, TC - total cholesterol, TG - triglyceride, HDL-C - high-density lipoprotein cholesterol, $L D L-C$ - low-density lipoprotein cholesterol, OGTT - oral glucose tolerance test. 
Table IV. Relationship between PM20D1 and poor pregnancy outcomes

\begin{tabular}{|lcccc|}
\hline Variables, $n$ (\%) & $\begin{array}{c}\text { GDM, PM20D1 high } \\
(n=99)\end{array}$ & $\begin{array}{c}\text { GDM, PM20D1 low } \\
(n=90)\end{array}$ & $\begin{array}{c}\text { Healthy control } \\
(n=100)\end{array}$ & $P$-value \\
\hline Premature & $4(4.04)$ & $10(11.11)$ & $2(2.00)$ & 0.014 \\
\hline Gestational hypertension & $8(8.08)$ & $14(15.56)$ & $4(4.00)$ & 0.016 \\
\hline Preeclampsia & $3(3.03)$ & $6(6.67)$ & $1(1.00)$ & 0.091 \\
\hline Hydramnios & $2(2.02)$ & $2(2.22)$ & $1(1.00)$ & 0.779 \\
\hline Fetal macrosomia & $4(4.04)$ & $5(5.56)$ & $3(3.00)$ & 0.662 \\
\hline Total & $21(21.21)$ & $37(41.11)$ & $11(11.00)$ & $<0.001$ \\
\hline
\end{tabular}

Table V. Risk factors for poor prognosis of pregnancy

\begin{tabular}{|lcccc|}
\hline $\begin{array}{l}\text { Factors at 24-28 weeks } \\
\text { of pregnancy }\end{array}$ & Wald & Odds ratio & $95 \% \mathrm{Cl}$ & $P$-value \\
\hline PM20D1 & 40.698 & -13.692 & $1.130(0.683-7.589)$ & $<0.001$ \\
\hline HOMA-IR & 0.214 & -0.471 & $0.623(0.084-4.586)$ & 0.643 \\
\hline FBG & 2.676 & 0.635 & $1.887(0.881-4.041)$ & 0.101 \\
\hline OGTT-1hPG & 7.898 & -1.909 & $0.148(0.039-0.561)$ & 0.004 \\
\hline OGTT-2hPG & 1.378 & 0.642 & $1.901(0.650-5.559)$ & 0.240 \\
\hline TG & 0.501 & -0.314 & $0.730(0.305-1.743)$ & 0.478 \\
\hline HDL-C & 0.452 & 1.010 & $2.746(0.144-52.228)$ & 0.501 \\
\hline LDL-C & 1.530 & -0.724 & $0.484(0.153-1.527)$ & 0.216 \\
\hline CRP & 0.114 & -0.040 & $0.959(0.757-1.215)$ & 0.734 \\
\hline IL-1 $\beta$ & 0.004 & -0.008 & $0.991(0.769-1.277)$ & 0.946 \\
\hline IL-6 & 0.167 & -0.060 & $0.941(0.705-1.256)$ & 0.682 \\
\hline TNF- $\alpha$ & 0.698 & 0.179 & $1.196(0.785-1.822)$ & 0.403 \\
\hline Leptin & 0.002 & 0.006 & $1.006(0.793-1.275)$ & 0.960 \\
\hline Adiponectin & 0.657 & 0.132 & $1.141(0.828-1.572)$ & 0.417 \\
\hline
\end{tabular}

$B M I$ - body mass index, FBG - fasting blood glucose, TC - total cholesterol, TG - triglyceride, HDL-C - high-density lipoprotein cholesterol, $L D L-C$ - low-density lipoprotein cholesterol, OGTT - oral glucose tolerance test, CRP - C-reactive protein, IL-1 $\beta$ - interleukin-1 $\beta$, IL-6 - interleukin-6, TNF- $\alpha$ - tumor necrosis factor $\alpha$.

gestational hypertension and total poor pregnancy rate were all remarkably higher in GDM patients with lower PM20D1 compared with other groups of patients (all $p<0.05$ ). As shown in Table $\mathrm{V}$, the logistic regression analysis showed that factors of PM20D1 and OGTT-1hPG at 24-28 weeks of pregnancy were independent risk factors for poor pregnancy outcomes.

\section{Discussion}

Gestational diabetes mellitus is one of the common complications during pregnancy. However, the mechanisms for GMD are still unclear and serum biomarkers for GDM are also inadequate. In this research, we demonstrated that serum PM20D1 was down-regulated in GMD patients and was associated with patients' clinical outcomes and pregnancy outcomes.

Many factors are reported to be involved in the GDM process $[14,15]$. In a recent report, Rancourt et al. found that levels of TNF- $\alpha$ and SOCS3 were significantly increased in visceral adipose tissues of GDM patients [16]. Cuili et al. showed that acti- vation of the AMPK signaling pathway in a mouse GDM model could decrease the inflammatory factors of IL-1 $\beta$, IL- 6 , and TNF- $\alpha$ and improve the GDM [17]. Another study also demonstrated that single nucleotide polymorphisms of IL- 6 and TNF- $\alpha$ were associated with incidence of GDM and serum inflammatory factor levels were generally higher in GDM patients than the healthy control [18]. In a more recent study, Alamolhoda et al. found that patients with higher CRP levels had significantly higher risk of developing GDM [19]. Also, studies also found that leptin was up-regulated and adiponectin was down-regulated in GDM patients and they had potential as diagnostic biomarkers [20-22]. In our research, we also found that the inflammatory factors were increased in GDM patients. However, to our best knowledge, no study has reported the role of PM20D1 in GDM.

PM20D1 is considered to be associated with lipid metabolism and obesity. Long et al. found that PM20D1 was a bidirectional enzyme in vitro and increased circulating PM20D1 augmented respiration and increased $\mathrm{N}$-acyl amino acids in blood 
[23]. Benson et al. found that genetic variation in PM20D1 expression was associated with obesity and might influence obesity treatment [24]. It was also found that a decreased level of PM20D1 might lead to white and brown adipose loss and reduced body weight [25]. Although studies on PM20D1 in pregnant women are rare, many studies have noted that lipid metabolism and obesity are closely associated with GDM. It was reported that maternal obesity and GDM were associated with decreased levels of genes involved in lipid metabolism, while adipokines such as TNF- $\alpha$, IL-1 $\beta$ and/or leptin were increased in adipose tissue of obese and GDM women, while the suggested dysfunction of lipid metabolism and activation of information might be associated with GDM and obesity in pregnant women [26]. Another study found that in GDM patients, $76.3 \%$ were overweight or obese, indicating that obesity was associated with GDM and was a risk factor for GDM [27]. All these studies implied that GDM was associated with lipid metabolism and obesity. Moreover, lipid metabolism was also associated with insulin resistance in GDM. It was also found that improvement of lipid metabolism could improve insulin resistance in GDM [28-30]. Thus, PM20D1 might influence GDM by regulating lipid metabolism, which needs more studies for confirmation [31]. In this research, we found that PM20D1 was down-regulated in GMD patients. We also found that in PM20D1 low expression patients, levels of TC and LDL-C were markedly higher, while levels of HDL-C were significantly lower, further indicating that PM20D1 was associated with lipid metabolism in GDM patients. Also, we observed that serum PM20D1 levels were the lowest at the second trimester of pregnancy, indicating that PM20D1 might be associated with pregnancy stage. However, deeper insights are still needed to illuminate role of PM20D1 in GMD or diabetes.

The present research also has some limitations. First, the study only contained 189 GDM patients. Secondly, we did not know the molecular mechanisms for PM20D1 in GDM. All these need more research.

In conclusion, this observational study demonstrated that PM20D1 was down-regulated in GDM patients and was associated with patients' clinical outcomes and pregnancy outcomes. This result might provide novel potential biomarkers for GDM, as well as novel research and treatment targets.

\section{Conflict of interest}

The authors declare no conflict of interest.

\section{References}

1. Goyal A, Gupta Y, Singla R, Kalra S, Tandon N. American Diabetes Association "Standards of Medical Care
- 2020 for Gestational Diabetes Mellitus": a critical appraisal. Diabetes Ther 2020; 11: 1639-44.

2. Plows JF, Stanley JL, Baker PN, Reynolds CM, Vickers MH. The pathophysiology of gestational diabetes mellitus. Int J Mol Sci 2018; 19: 3342.

3. McIntyre HD, Catalano P, Zhang C, Desoye G, Mathiesen ER, Damm P. Gestational diabetes mellitus. Nat Rev Dis Primers 2019; 5: 47.

4. Johns EC, Denison FC, Norman JE, Reynolds RM. Gestational diabetes mellitus: mechanisms, treatment, and complications. Trends Endocrinol Metab 2018; 29: 743-54.

5. Chiefari E, Arcidiacono B, Foti D, Brunetti A. Gestational diabetes mellitus: an updated overview. J Endocrinol Invest 2017; 40: 899-909.

6. Eades CE, Cameron DM, Evans JMM. Prevalence of gestational diabetes mellitus in Europe: a meta-analysis. Diabetes Res Clin Pract 2017; 129: 173-81.

7. Damm P, Houshmand-Oeregaard A, Kelstrup L, Lauenborg J, Mathiesen ER, Clausen TD. Gestational diabetes mellitus and long-term consequences for mother and offspring: a view from Denmark. Diabetologia 2016; 59 : 1396-9.

8. Kim JT, Jedrychowski MP, Wei W, et al. A plasma protein network regulates PM20D1 and N-acyl amino acid bioactivity. Cell Chem Biol 2020; 27: 1130-9.

9. Sanchez-Mut JV, Heyn H, Silva BA, Dixsaut L, Johannes G. PM20D1 is aquantitative trait locus associated with Alzheimer's disease. Nat Med 2018; 24: 598-603.

10. Long JZ, Roche AM, Berdan CA, et al. Ablation of PM20D1 reveals $\mathrm{N}$-acyl amino acid control of metabolism and nociception. Proc Natl Acad Sci USA 2018; 115: E6937-45.

11. Kim JT, Terrell SM, Li VL, Wei W, Fischer CR, Long JZ. Cooperative enzymatic control of $\mathrm{N}$-acyl amino acids by PM20D1 and FAAH. Elife 2020; 9: e55211.

12. Gunawardhana LP, Baines KJ, Mattes J, Murphy VE, Simpson JL, Gibson PG. Differential DNA methylation profiles of infants exposed to maternal asthma during pregnancy. Pediatr Pulmonol 2014; 49: 852-62.

13. Wendland EM, Torloni MR, Falavigna M, et al. Gestational diabetes and pregnancy outcomes - a systematic review of the World Health Organization (WHO) and the International Association of Diabetes in Pregnancy Study Groups (IADPSG) diagnostic criteria. BMC Pregnancy Childbirth 2012; 12: 23.

14. Bellia C, Zaninotto M, Cosma C, et al. Definition of the upper reference limit of glycated albumin in blood donors from Italy. Clin Chem Lab Med 2017; 56: 120-5.

15. Giglio RV, Lo Sasso B, Agnello L, et al. Recent updates and advances in the use of glycated albumin for the diagnosis and monitoring of diabetes and renal, cerebro- and cardio-metabolic diseases. J Clin Med 2020; 9: 3634.

16. Rancourt RC, Ott R, Ziska T, et al. Visceral adipose tissue inflammatory factors (TNF-alpha, SOCS3) in gestational diabetes (GDM): epigenetics as a clue in GDM pathophysiology. Int J Mol Sci 2020; 21: 479.

17. Zou C, Zhang Q, Zhang S. Mogroside IIIE attenuates gestational diabetes mellitus through activating of AMPK signaling pathway in mice. J Pharmacol Sci 2018; 138: 161-6.

18. Zhang J, Chi H, Xiao H, et al. Interleukin 6 (IL-6) and tumor necrosis factor $\alpha$ (TNF- $\alpha$ ) single nucleotide polymorphisms (SNPs), Inflammation and metabolism in gestational diabetes mellitus in Inner Mongolia. Med Sci Monit 2017; 23: 4149-57.

19. Alamolhoda SH, Yazdkhasti M, Namdari M, Zakariayi SJ, Mirabi P. Association between C-reactive protein 
and gestational diabetes: a prospective study. J Obstet Gynaecol 2020; 40: 349-53.

20. Lekva T, Michelsen AE, Pål A, Henriksen T, Ueland T. Leptin and adiponectin as predictors of cardiovascular risk after gestational diabetes mellitus. Cardiovasc Diabetol 2017; 16: 5.

21. Thagaard INS, Krebs L, Holm JC, Lange T, Larsen T, Christiansen $M$. Adiponectin and leptin as first trimester markers for gestational diabetes mellitus: a cohort study. Clin Chem Lab Med 2017; 55: 1805-12.

22. Pawlik A, Teler J, Maciejewska A, Sawczuk M, Safranow K, Dziedziejko V. Adiponectin and leptin gene polymorphisms in women with gestational diabetes mellitus. J Assist Reprod Genet 2017; 34: 511-6.

23. Long JZ, Svensson KJ, Bateman LA, Hua L, Bruce MS. The secreted enzyme PM20D1 regulates lipidated amino acid uncouplers of mitochondria. Cell 2016; 166: 424-35.

24. Benson KK, Hu W, Weller AH, Bennett AH, Soccio RE. Natural human genetic variation determines basal and inducible expression of PM20D1, an obesity-associated gene. Proc Natl Acad Sci USA 2019; 116: 23232-42.

25. Li D, Liu Y, Gao W, Han J, Pang W. Inhibition of miR-324$5 p$ increases PM20D1-mediated white and brown adipose loss and reduces body weight in juvenile mice. Eur J Pharmacol 2019; 863: 172708.

26. Lappas M. Effect of pre-existing maternal obesity, gestational diabetes and adipokines on the expression of genes involved in lipid metabolism in adipose tissue. Metabolism 2014; 63: 250-62.

27. Black MH, Sacks DA, Xiang AH, Lawrence JM. The relative contribution of prepregnancy overweight and obesity, gestational weight gain, and IADPSG-defined gestational diabetes mellitus to fetal overgrowth. Diabetes Care 2013; 36: 56-62.

28. Stein S, Stepan H, Kratzsch J, et al. Serum fibroblast growth factor 21 levels in gestational diabetes mellitus in relation to insulin resistance and dyslipidemia. Metabolism 2010; 59: 33-7.

29. Tumurbaatar B, Poole AT, Olson G, et al. Adipose tissue insulin resistance in gestational diabetes. Metab Syndr Relat Disord 2017; 15: 86-92.

30. Butte NF. Carbohydrate and lipid metabolism in pregnancy: normal compared with gestational diabetes mellitus. Am J Clin Nutr 2000; 71: 1256S-61S.

31. Huang $L$, Yue $P, W u X$, et al. Combined intervention of swimming plus metformin ameliorates the insulin resistance and impaired lipid metabolism in murine gestational diabetes mellitus. PLoS One 2018; 13: e0195609. 